\title{
Assessment of DC/DC Converters for use in DC Nodes for Offshore Grids
}

\author{
C. E. Sheridan*, M.M.C. Merlin*, T. C. Green* \\ *Imperial College London, UK, \{c.sheridan11 michael.merlin07,t.green\}@imperial.ac.uk
}

Keywords: HVDC, DC/DC converter, offshore grid, DC node

\begin{abstract}
With increasing offshore wind generation, there is a strong argument for implementing a multi-terminal DC grid offshore by the interconnection of individual HVDC links. The point of intersection of three or more lines can be used to interconnect projects with different voltage levels and to control power distribution. It is being proposed that these points, or nodes, be implemented using DC devices. A highvoltage, high-power DC/DC converter will therefore be an important component of a DC node. This paper reviews possible DC/DC converter topologies, looking at device requirement, different voltage conversion ratio and fault management. The suitability of the converters considered, for use in a node in a HVDC offshore grid, is discussed. A resonant $\mathrm{DC} / \mathrm{DC}$ converter topology is considered in detail and is modelled at a conversion ratio of two, and demonstrates high power efficiency.
\end{abstract}

\section{Introduction}

An offshore grid in the North Sea has the potential to increase the penetration of renewable energy in Europe and to improve international energy transfer between European countries [1]. It has been long acknowledged that this offshore grid could be considered as a precursor to a European SuperGrid. It has also been proposed that this SuperGrid could connect the offshore wind generation, from the North Sea, to the potential solar generation in southern Europe, the geothermal generation in Iceland, and the hydro generation in Norway [2]. It has been well established that an offshore grid will be implemented using HVDC transmission, as it is more efficient and costeffective than HVAC when using submarine cables. Furthermore Voltage Source Converter (VSC) technology is more suitable for multi-terminal HVDC (MTDC) networks as reversing the power flow is done by changing current polarity, whereas classic HVDC (line commutated converter) does this by reversing voltage polarity. The main restriction with realising a DC grid has been the lack of a commercially available DC circuit breaker [3], [4], although one has been proposed, and tested at $80 \mathrm{kV}$, [5]. There have also been VSC converters presented that have the ability to block DC faults [6], [7].

As of yet there is no established grid code for an offshore grid, and as cable ratings rise by tens of kilovolts at each iteration of the technology, wind farms that are constructed adjacent to one another could have different voltage levels. For example, if two wind farms projects are constructed near each other, at a sufficient distance that would necessitate the use of DC transmission but are not constructed at the same voltage level, it would not be possible to connect these wind farms directly. This might be the case in some of the Round 3 Crown Estate zones in UK waters [8], because these projects may not be constructed at the same time and may therefore, use converters and cables from different iterations of the technology.

Nodes, the points of intersection of three or more lines, will be an important element of DC grids, as they can be used to join paths of the grid together and to control the distribution of power. Before a node topology can be discussed the technologies and components that could potentially be used should be identified. A DC/DC converter is analogous to the transformer in an AC transmission system; it allows DC systems of different voltage levels to be interconnected. They could also allow systems from differing manufacturers to be connected and allow interconnection between classic HVDC systems, and VSC systems. There is also the opportunity to create DC collection systems for wind farms [9]. The output from a group of wind turbines goes through several power conversion stages and having a simpler power chain, that avoids using $\mathrm{AC} / \mathrm{DC}$ conversion, and use $\mathrm{DC} / \mathrm{DC}$ converters to step-up to the collection network voltage and be more efficient and more cost effective.

It has been shown that a single converter topology cannot be used for all applications [10]. This paper will give an overview of DC/DC converters, looking at several topologies. A resonant $\mathrm{DC} / \mathrm{DC}$ converter is then examined in greater detail. The focus of the paper is to examine converters that would be suitable for use in a node in a MTDC offshore grid.

\section{DC/DC Converters}

There are two ways of interfacing DC systems: a) use two $\mathrm{AC} / \mathrm{DC}$ converters front-to-front resulting in a $\mathrm{DC} / \mathrm{AC} / \mathrm{DC}$ converter, shown in Figure 1, or b) using a dedicated DC/DC converter, like the ones shown in Figures 2, 3 and 4.

The use of an intermediate AC stage in a DC/DC converter improves the converters robustness, as DC faults can be blocked by bringing the AC voltage to zero. Often, AC transformers are used (possibly at high frequency) in DC/DC conversion as they enable higher conversion ratios with 
relatively low losses. Many transformer-less topologies make use of switched capacitors or inductors. These circuits can be modular in design and thus are easily scalable. However, due to high amplitude current spikes when charging the capacitors of switched-capacitor circuits, these topologies are not generally suited to high-power, high-voltage applications. The following subsections will examine different topologies for high-voltage, high-power DC/DC converters.

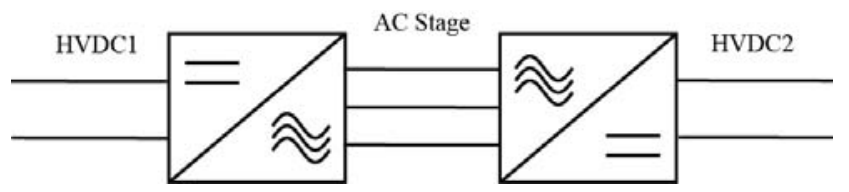

Figure 1: DC/AC/DC converter comprised of two AC/DC converters

\subsection{Front-to-Front DC/AC/DC Converter}

There are several DCAC/DC converters proposed in [10], with the first one presented being a converter based upon the multi-level VSC topology from [6], the Alternate Arm Converter (AAC). The AAC is modular, providing low effective switching rates, redundancy and increased reliability. Each module can be a full $\mathrm{H}$-bridge or a half $\mathrm{H}$ bridge cell, an example of these cells is shown in Figure A.1 in the Appendix. The choice affects the ability to protect against DC faults and the conduction power loss. The obtained DC/AC/DC converter comprises two 3-phase AACs directly coupled through inductors. As the AC connection is independent of the AC grid, it can be optimised in terms of $\mathrm{AC}$ voltage level such that the DC connections can operate at their optimum point and reduce power losses. However, as the converter is directly linked, high ratio voltage conversion is not achievable, as the two AACs would run away from their respective optimum points resulting in unacceptable losses. A low ratio conversion (less than two) could be practical for DC interconnection in an offshore DC node. Given that cable ratings increase by increments of tens of kilovolts, wind farm projects a few years apart may be of a similar but not common voltage level. The converter described above could be used to interconnect these wind farms and also provide DC-fault blocking capabilities.

\subsection{Transformer -Coupled DC/AC/DC Converter}

The second converter presented in [10], is a transformer coupled DC/AC/DC converter, also based on the AAC. It has a similar configuration to the converter discussed in Section 2.1 , with the main difference being that the AC connection is a single phase transformer rather than 3-phase. This topology also uses cells which can be full or half $\mathrm{H}$-bridge configuration. The AC transformer is independent of any AC grid, implying that it can be designed for higher frequencies, so reducing the volume of the transformer, and the $\mathrm{AC}$ voltage and current does not need to be sinusoidal. The use of the transformer provides galvanic separation between the DC systems, and should be insulated to withstand the DC voltage stress in the instance of a DC fault. Also transformer coupling enables a higher conversion ratio than the front-to-front converter. It could be used to interconnect DC systems of differing voltage levels that require a higher conversion ratio, than the converter in Section 2.1 can provide, since each AAC can be operated closest to its own optimum operating point. This could allow this topology to be used to step up the voltage of a DC collector system to a HVDC network.

\subsection{LCC/VSC DC/AC/DC Converter}

Another interesting converter proposed in [10], is one that would enable interconnection between classic linecommutated converters (LCCs) and VSCs. The converter has a DC/AC/DC format, coupled using a 3-phase transformer. Like the previous two topologies this converter is modular using full $\mathrm{H}$-bridge cells. The AC link for this converter can be designed to operate at a higher frequency and the $\mathrm{AC}$ voltage and current need not be sinusoidal. The converter is able to maintain the correct DC voltage polarity while allowing power exchange between the two systems. It could be used to connect an older LCC wind farm project with a newly constructed, nearby VSC project. This could allow existent LCC based wind farms to be incorporated into a MTDC grid.

\subsection{Multiple-Module DC/DC Converters}

Two multiple-module converters are presented in [11]. They use single-switch, single-inductor modules. A cascade connected and series connected converter are described. A simple block diagram of the arrangements is shown in Figure 2. Both converters use high power semiconductor devices for the switches. The cascade converter is the connection of two conventional boost converters one after another, the output of the first boost converter is connected directly to the input of the second, implying that both must be rated for the full input power. The advantage of this arrangement is that through this cascaded connection, high conversion ratios can be achieved as the gains of the two converters are multiplied together. However the input power must be processed by converters, meaning significant losses from two sets of conduction and switching power loss.

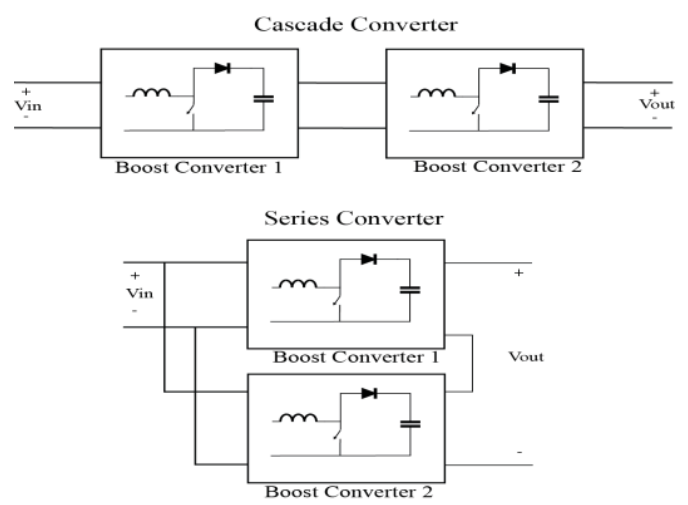

Figure 2: Block diagram of converters proposed in [11] 
The series connected converter is the connection of two boost converters with their inputs connected in parallel and their outputs connected in series. This connection requires care so as not to create a short-circuit of the negative rail to the source. The connection of the series converter is done by swapping the position of the inductor and reversing the diode direction in Boost Converter 2, as shown in the Appendix in Figure A.2. This topology cannot achieve such high-gains as the cascaded topology because the gains of the individual converters are added together, and not multiplied. Both modules see the full input voltage but only process half of the input power. For a node in a HVDC grid, the requirement of high conversion ratios may not be needed but given that these converters have unidirectional power flow their application may be restricted. In [11] the experimental results for scaled down prototypes demonstrated efficiency in the range of $71 \%$ to $87 \%$. Such low efficiencies would make these converters unsuitable for use in an HVDC grid.

\subsection{Modular Resonant DC/DC Converter}

A family of modular active switched DC/DC converters is presented in [12]. The topology consists of a resonant inductor with actively switched capacitors which are arranged in stacks of H-bridge cells. Being modular implies easier design of large converters and increased reliability, through the inclusion of redundant modules. By rotating the capacitors, a resonance between the capacitors and the inductors can be induced. Then by controlling the synchronisation between the rotation of the capacitor and the induced oscillation, the power transfer and stepping ratio can be adjusted. The converters are operated in discontinuous conduction mode (DCM) in order to achieve soft-switching of the semiconductor devices (and thereby reduce the switching power loss). The resonance of the LC circuit in the converter implies natural commutation, thus allowing thyristors to be used as the semiconductor devices. The step-up, step-down, and inverting converters discussed here only allow unidirectional power flow. A bidirectional converter is described which is a hybrid of the step-up and step-down converters, and is shown in Figure 3.

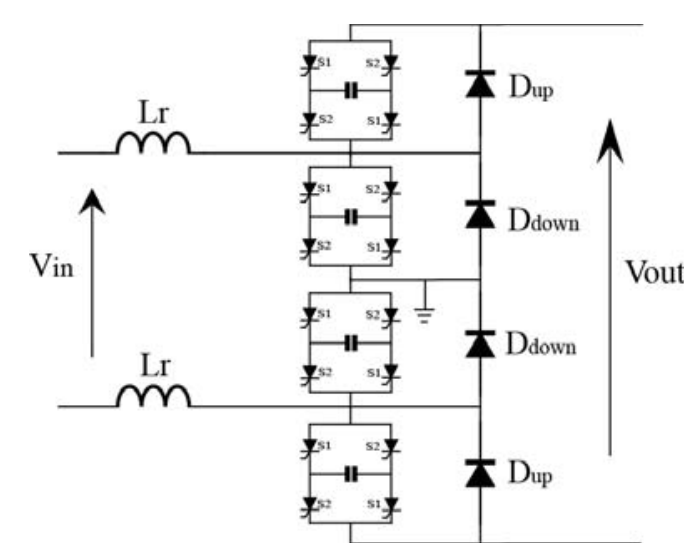

Figure 3: Bidirectional modular resonant converter

The circuit can be designed with two inductors, one for stepup operation and the other for step-down, or with a single inductor for both operating conditions. An inverting bidirectional converter is also described. It can be seen from the circuit diagram that a short-circuit fault on the output terminals, can propagate through the converter, but a fault on the input terminals can be blocked by the diode and by terminating the gate signal of the thyristors.

An experimental $5 \mathrm{~kW}$ prototype was built and for step-up operation exhibited losses of $88.7 \%$ and for the step converter efficiency figures of $95.5 \%$ were recorded. The low efficiency figure would suggest that the step-down converter is unsuitable for DC grid use. The step-up converter could be used to connect a DC collection network with the HVDC grid.

\section{Resonant DC/DC Converter}

\subsection{Converter Operation}

A bidirectional resonant DC/DC converter has been proposed in [13], the circuit is shown in Figure 4. This circuit uses LC resonance to transfer charge to the intermediary capacitor and then onward to the output.

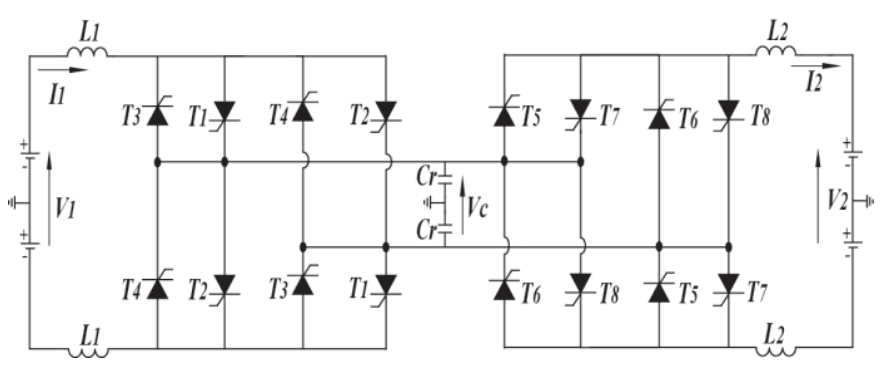

Figure 4: Bidirectional Resonant DC/DC Converter

The polarity of the voltage across the capacitor is rotated by alternately switching the semiconductors devices. Power flow through the circuit is reversible by changing the current polarity, making it suitable for use with VSC technology. This converter is also able to provide negative voltage and/or current on either terminal [13]. The resonant action creates regular zero-crossings of the current, and so thyristors can be used as the switching devices. The use of thyristors implies fewer semiconductor devices need to be used (because of the higher voltage ratings available for thyristors compared to IGBTs) and thus lower conduction losses. The converter is operated in DCM which provides soft-switching of the thyristors and relatively low switching power loss. The switching frequency is used to control the circuit, and a phase-locked-loop is used to synchronise the frequency to the approximate sinusoidal voltage, $V_{c}$, across $C_{r}$. It can be seen from the literature for this family of resonant converters, [1315] that the lower the conversion ratio, the higher the efficiency of the converter.

It has been shown in [14] that this converter has DC fault blocking capability, and controllable operation during faults. The thyristors on the lower-voltage side must be rated to the 
voltage on the higher-voltage side in order to improve fault tolerance. Controlled operation is achieved by reducing the switching frequency of the converter. From the analysis provided in [13], it can be seen that the switching frequency, $f_{s}$, is related to the terminal voltages by Equation (1).

$$
f_{s}=\frac{I_{2}\left(V_{2}-V_{1}\right)}{2 C_{r} V_{2} V_{1}}
$$

This relationship implies that the closer $V_{1}$ comes to $V_{2}$ that the frequency will reduce. It can also be seen from the literature that the conversion ratio should meet the condition expressed in Equation (2), to fully ensure its fault blocking capabilities for all scenarios.

$$
\frac{V_{1}}{V_{2}-V_{1}} \leq 1
$$

The analysis implies that the conversion ratio should be two or greater, so that the turn-off time for the circuit is not lower than the extinction time of the selected thyristors. In thyristor datasheets the turn-off time for the device is specified, usually as $T_{q}$, implying the turn-off time for the circuit must always be greater than $T_{q}$. This is related to the switching frequency shown in Equation (3). If this condition is not met, should a fault occur and the frequency increases beyond $f_{\text {smax }}$, the thyristor current will not have time to fall below the holding current of the device and it will remain latched on.

$$
f_{\text {smax }}=\frac{1}{2 T_{q}}
$$

\subsection{Simulation and Results}

An $80 \mathrm{kV}$ to $160 \mathrm{kV}, 100 \mathrm{MW}$ converter was simulated in MATLAB Simulink, using the SimPowerSystems toolbox. The parameters for the converter, and the thyristor device, are given in the Appendix. The thyristor device used was ABB's 5STP 21H4240. Some example voltage and current waveforms for the circuit can be seen in Figure 5 and Figure 6. These waveforms show converter start up with steady state reached after $0.4 \mathrm{~s}$.
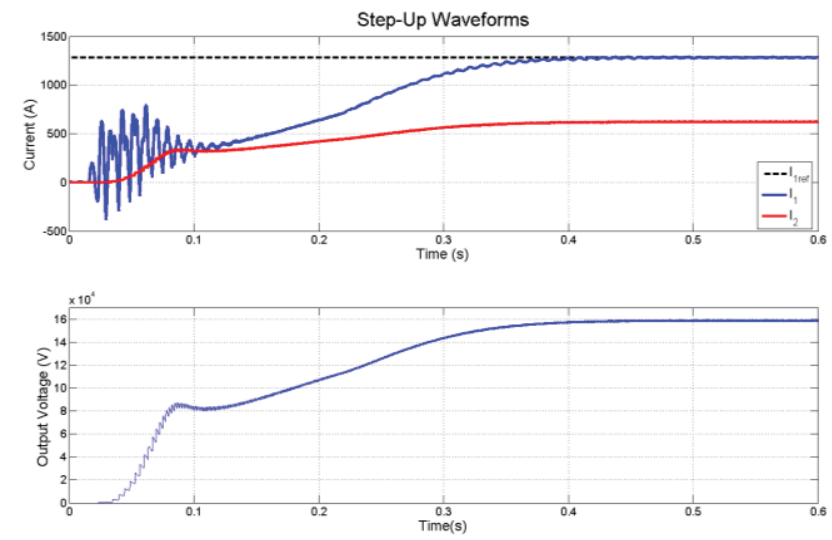

Figure 5: Step-up current and voltage waveforms
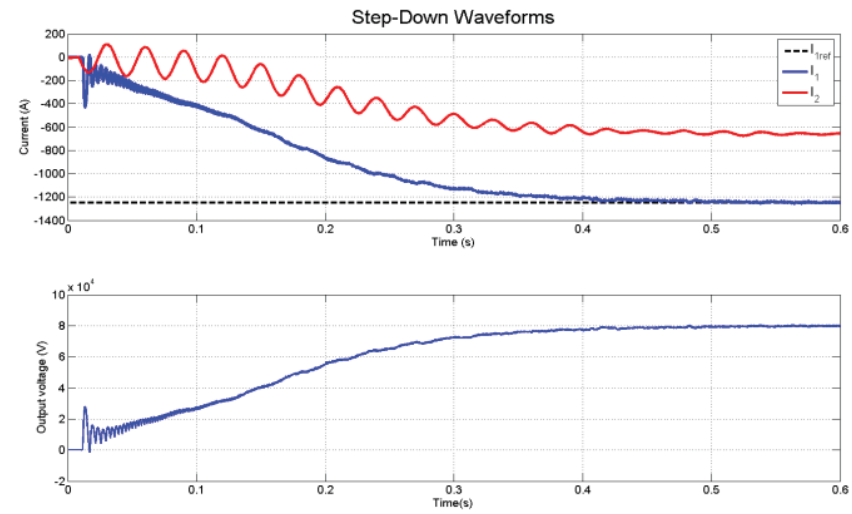

Figure 6: Step-down current and voltage waveforms

This model was used to evaluate the losses of the converter. The conduction losses were calculated by post-processing the current waveforms of the thyristors from the Simulink model. Each thyristor is modelled as a fixed on-state voltage plus a resistance and so, the average power loss was found by integrating the instantaneous current waveforms of the thyristor and then using Equation (4) to determine the conduction losses.

$$
P_{\text {cond }}=V_{\text {on }} I_{\text {avg }}+R_{\text {on }} I_{r m s}^{2}
$$

Figure 7 shows an example of the current across the thyristor in steady state. The RMS and average of these current waveforms were used in Equation (4) above to find the conduction losses of the thyristor.

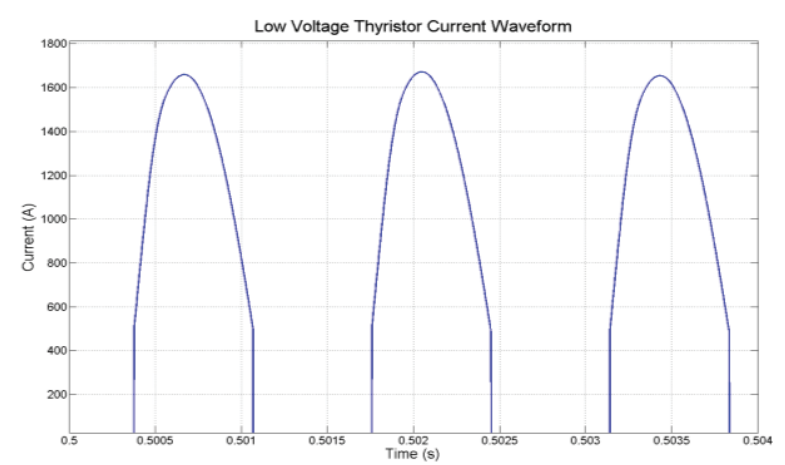

Figure 7: Current waveforms of the low voltage thyristors

Given that the circuit is soft-switched this implies that the thyristors turn off at zero voltage and turn on at zero current. This suggests negligible switching losses. The study of semiconductor losses of the modular resonant converter from [12] shows that conduction losses dominate device losses.

\begin{tabular}{|l|c|c|}
\cline { 2 - 3 } \multicolumn{1}{c|}{} & $\begin{array}{c}\text { LV Side } \\
\text { (kW) }\end{array}$ & $\begin{array}{c}\text { HV Side } \\
\text { (kW) }\end{array}$ \\
\hline Conduction & 321 & 192 \\
\hline Total (kW) & \multicolumn{2}{|c|}{513} \\
\hline
\end{tabular}

Table 1: Semiconductor losses 
From this it can be seen that the converter has an efficiency of $99.48 \%$, at a conversion ratio of two, for a $100 \mathrm{MW}$ converter.

\subsection{Discussion}

Several results have been published for the efficiency of this resonant DC/DC converter [13-15] and these have been plotted in Figure 7 as a function of voltage conversion ratio (high-side voltage to low-side voltage). It is clear that the efficiency improves in an approximately linear fashion as the ratio approaches unity. A further detailed parametric analysis of this converter is required to determine more accurate efficiency as a function of design point and operating point.

The same papers also suggest that the controllability of the converter under fault conditions could be jeopardised for conversion ratios of less than two (because of encroachment on the extinction time of the thyristors). This would appear to limit application of the converter and may exclude it from the role of interfacing HVDC links of similar but different nominal voltage. This converter could be used as an interface between a DC collection network of a wind farm and an HVDC route. However, for large ratios (beyond, say, 10) it is likely to be more efficient to use a transformer-coupled $\mathrm{DC} / \mathrm{AC} / \mathrm{DC}$ converter.

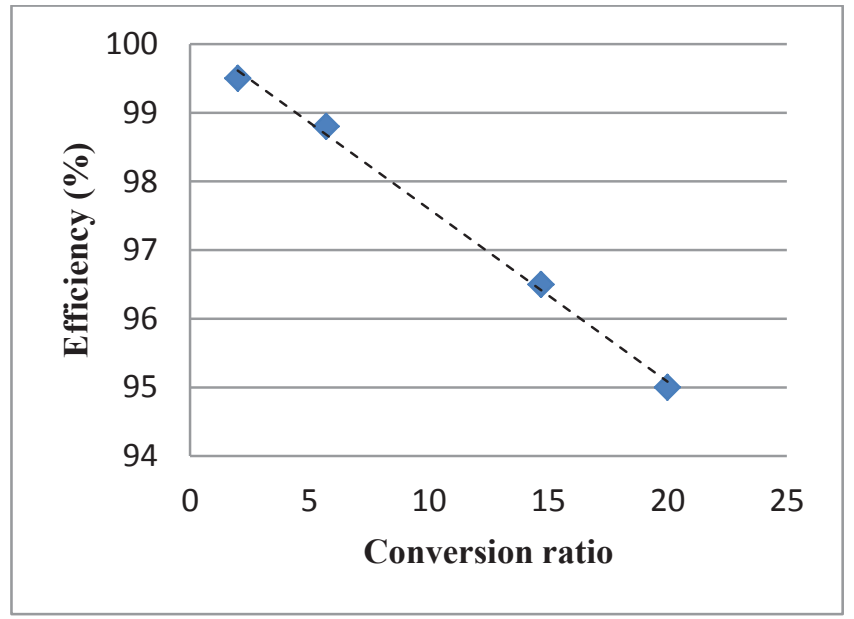

Figure 8: Conversion ratio versus efficiency [13-15]

\section{Conclusion}

This paper has presented the need for DC/DC converters in a DC node in an offshore HVDC grid. A converter with a conversion ratio close to unity could be used to interconnect adjacent wind farms that operate at close, but not identical, voltage levels. If the node also connects to the DC collection network of a wind farm then voltage conversion ratios of greater than 10 may be needed. Several converters were reviewed and their suitability for the two cases assessed. Of the converters considered, a resonant DC/DC converter was studied in detail. This converter is not suitable for use for connecting systems of similar voltages but would be more appropriate for interfacing wind farm collection systems.

\section{Acknowledgements}

The authors gratefully acknowledge the financial support of the Research Councils UK through the HubNet Project (grant number: EPI013636/1), and would like to thank Mr Kamal Siriwardhana of Arup, Transmission and Distribution for his discussion of node design issues.

\section{Appendix}

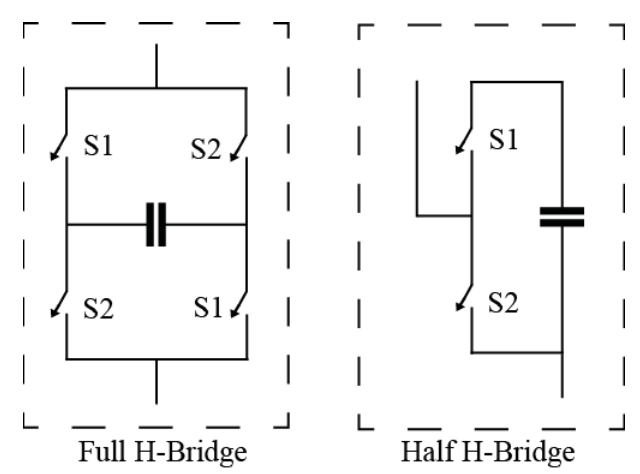

Figure A.1: Full and half H-bridge configuration

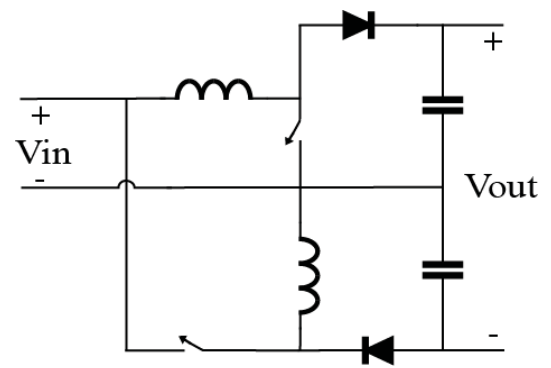

Figure A.2: Series module converter circuit diagram

\begin{tabular}{|c|c|c|}
\hline Component & Value & Unit \\
\hline$P_{a v}$ & 100 & $\mathrm{MW}$ \\
\hline$V_{\text {low }}$ & 80 & $\mathrm{kV}$ \\
\hline$V_{\text {high }}$ & 160 & $\mathrm{kV}$ \\
\hline$f_{s}$ & 720 & $\mathrm{~Hz}$ \\
\hline$f_{\text {smax }}$ & 1250 & $\mathrm{~Hz}$ \\
\hline$C_{r}$ & 4 & $\mu \mathrm{F}$ \\
\hline$L_{1}$ & 25 & $\mathrm{mH}$ \\
\hline$L_{2}$ & 1 & $\mathrm{mH}$ \\
\hline$R_{\text {on }}$ & $0.191 \times 83$ & $\mathrm{~m} \Omega$ \\
\hline$V_{\text {on }}$ & $1.249 \times 83$ & $\mathrm{~V}$ \\
\hline
\end{tabular}

Table A.1: Resonant DC/DC converter parameters

\section{References}

[1] "North Sea Countries' Offshore Grid Initiative Memorandum of Understanding." European Commission, 2010. 
[2] D. Van Hertem, M. Ghandhari, and M. Delimar, "Technical limitations towards a SuperGrid - A European prospective," in 2010 IEEE International Energy Conference, 2010, pp. 302-309.

[3] D. Van Hertem, M. Ghandhari, J. Curis, O. Despouys, and A. Marzin, "Protection requirements for a multiterminal meshed DC grid," Cigre Conference Bologna 2011, pp. 1-4.

[4] C. M. Franck, "HVDC Circuit Breakers: A Review Identifying Future Research Needs," IEEE

Transactions on Power Delivery, vol. 26, no. 2, pp. 998-1007, Apr. 2011.

[5] J. Häfner and B. Jacobson, "Proactive Hybrid HVDC Breakers - A key innovation for reliable HVDC grids," The electric power system of the future Integrating supergrids and microgrids International symposium - Cigré, 2011.

[6] M. M. C. Merlin, T. C. Green, P. D. Mitcheson, D. R. Trainer, D. R. Critchley, and R. W. Crookes, "A new hybrid multi-level voltage-source converter with DC fault blocking capability," in 9th IET International Conference on AC and DC Power Transmission (ACDC 2010), 2010, pp. 1-5.

[7] D. R. Trainer, C. C. Davidson, C. D. M. Oates, D. R. Macleod, D. R. Critchley, and R. W. Cookes, "A new Hybrid Voltage-Sourced Converter for HVDC Power Transmission," Cigre Session 2010, 2010.

[8] The Crown Estate, "UK Offshore Wind Report $2012, " 2012$.

[9] C. Zhan, C. Smith, a. Crane, a. Bullock, and D. Grieve, "DC transmission and distribution system for a large offshore Wind Farm," 9th IET International Conference on AC and DC Power Transmission (ACDC 2010), pp. O46-O46, 2010.

[10] C. D. Barker, C. C. Davidson, D. R. Trainer, and R. S. Whitehouse, "Requirements of DC-DC Converters to facilitate large DC Grids," in Cigre Session 2012, 2012.

[11] N. Denniston, A. M. Massoud, S. Ahmed, and P. N. Enjeti, "Multiple-Module High-Gain High-Voltage DC-DC Transformers for Offshore Wind Energy Systems," IEEE Transactions on Industrial Electronics, vol. 58, no. 5, pp. 1877-1886, May 2011.

[12] H. Abdelrahman, "A New Family of Transformerless Modular DC-DC Converters for High Power Applications," University of Toronto, 2011.
[13] D. Jovcic, "Bidirectional, high-power DC transformer," Power Delivery, IEEE Transactions on, vol. 24, no. 4, pp. 2276-2283, 2009.

[14] D. Jovcic and B. Wu, "Fast Fault Current Interruption on High-Power DC Networks," Power and Energy Society General Meeting,, pp. 4-9, 2010.

[15] D. Jovcic, "High-power, resonant DC/DC converter for integration of renewable sources," PowerTech, 2009 IEEE Bucharest, pp. 1-6, 2009. 